\title{
Sonochemical Coating of Cotton and Polyester Fabrics with "Antibacterial" BSA and Casein Spheres**
}

\author{
Ulyana Shimanovich, ${ }^{[a]}$ Artur Cavaco-Paulo, ${ }^{[\mathrm{b}]}$ Yeshayahu Nitzan, ${ }^{[\mathrm{c}]}$ and \\ Aharon Gedanken* ${ }^{[a]}$
}

\begin{abstract}
A novel antibacterial coating for cotton and polyester fabrics has been developed by using drug-loaded proteinaceous microspheres made of bovine serum albumin and casein proteins. The microbubbles were created and anchored onto the fabrics (see figure) in a one-step reaction that lasts $3 \mathrm{~min}$. The sonochemically produced "antibacterial fabrics" have been characterized. The efficiency of the sonochemical process in converting the native proteins into microspheres, encapsulating the drug, and coating the fabric has also been studied.
\end{abstract}

Keywords: antibacterial agents biological activity $\cdot$ proteins $\cdot$ sonochemistry

\section{Introduction}

The transmission of infectious disease agents in healthcare facilities is an increasingly important concern for both medical providers and the public. ${ }^{[1-4]}$ Microorganisms survive on textile materials and plastics ${ }^{[3]}$ and their transmission has been demonstrated to occur by surface contact with hands, fabrics, and hospital devices. ${ }^{[5,6]}$ There is an increasing need to develop novel and effective antimicrobial products that can combat infections and be applied to potentially contaminated wounds. A wide range of materials, including antibiotics, ${ }^{[7]}$ metal ions, ${ }^{[8]}$ and quaternary ammonium compounds, ${ }^{[9,10]}$ are known to prevent the attachment and proliferation of microbes on material surfaces.

Several methods for the production of antimicrobial coatings have been developed. The methods include sol-gel coating, ${ }^{[11]}$ spray and foam techniques, ${ }^{[12]}$ infra-red heating, agglutination, the use of adhesive compounds that improve the bonding of threads to rubber and proteins, and proteins

[a] U. Shimanovich, Prof. A. Gedanken

Department of Chemistry and Kanbar Laboratory for Nanomaterials Bar-Ilan University Center for Advanced Materials and Nanotechnology, Bar-Ilan University, Ramat-Gan 52900 (Israel) Fax: (+972)3-7384053

E-mail:gedanken@mail.biu.ac.il ushimanovich@yahoo.com

[b] Prof. A. Cavaco-Paulo

Textile Engineering Department, University of Minho 4800058 Guimaraes (Portugal)

[c] Prof. Y. Nitzan

Mina and Everard Goodman Faculty of Life Sciences Bar-Ilan University, Ramat-Gan 52900 (Israel)

[**] BSA = bovine serum albumin or polysaccharides with organosiloxane side-chains that bond to threads.

We have recently developed a novel method for imparting antimicrobial functions on cotton and polyester fabrics. ${ }^{[13,14]}$ The method employs sonochemical radiation as a technique for attaching drug-loaded proteinaceous microspheres onto cotton and polyester fabrics. ${ }^{[15]}$ Bovine serum albumin (BSA) and casein proteins were used to coat all the fabrics tested within this research. The formation, characterization, and properties of the sonochemically made proteinaceous microspheres (PMs) have recently been reviewed. ${ }^{[16]}$ According to the mechanism proposed for the sonochemical formation of PMs, the spheres are formed by chemically cross-linking the cysteine residues of the protein with the $\mathrm{HO}_{2}$ radical formed around a micron-sized gas bubble or nonaqueous droplet. ${ }^{[17]}$ The chemical cross-linking is responsible for the formation of the spheres and is a direct result of the chemical effects of ultrasound radiation on the aqueous medium. The microbubbles are created in a short process that lasts 3 min. ${ }^{[18]}$ Herein, an antibiotic drug, tetracycline (TTCL) ${ }^{[19-22]}$ was encapsulated in BSA or casein microspheres. The TTCL antibiotic has a broad range of activity, is relatively safe, can be used by many routes of administration, and is widely used. This paper describes the possibility of building a protective and wound-healing device that is capable of delivering the drug at the rate and for the time needed to accomplish the medical function. The perspective is to use these coated fabrics, inter alia, as antibacterial bandages in patients susceptible to contamination.

\section{Results and Discussion}

"Antibacterial fabrics" were prepared by sonochemically coating cotton and polyester fabrics with TTCL-loaded proteinaceous microspheres. An organic solvent, such as dodecane, was used as the co-solvent and the sonochemical pro- 
cess was performed for only $3 \mathrm{~min}$. The creation and attachment of TTCL-loaded PMs were achieved in a one-step process.

The morphologies of the cotton and polyester bandages coated with TTCL-loaded BSA or casein microspheres were determined by light microscopy. For both proteins (BSA and casein), the morphologies of the attached PMs were found to be similar. Figure 1a shows the image of an uncoat-
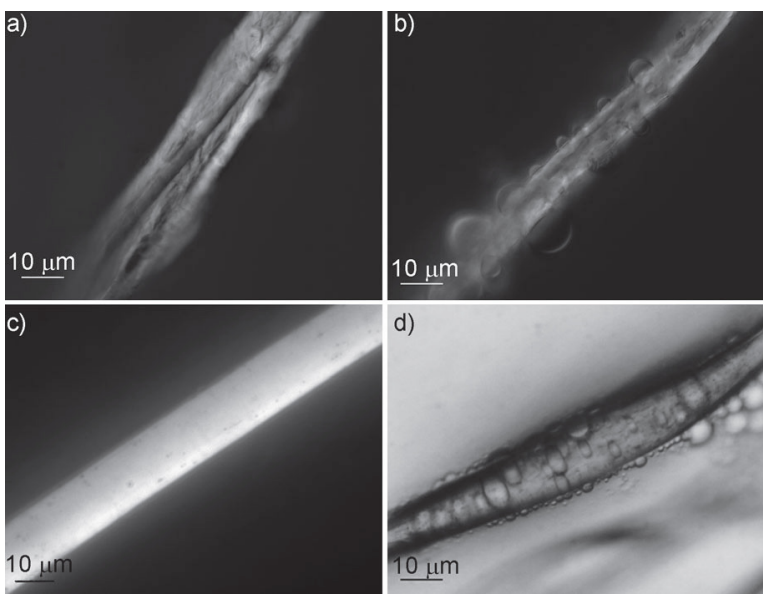

Figure 1. Apo-Tome images of a) a pristine cotton fiber, b) a cotton fiber coated with BSA microspheres, c) a pristine polyester fiber, and d) a polyester fiber coated with BSA spheres.

ed yarn of cotton. The fibrous nature of the yarn is clearly observed. In Figure 1b we observe many microspheres attached to the cotton fiber. Figure $1 \mathrm{c}$ and d show an uncoated and coated polyester fiber, respectively. Similar results were observed after coating the cotton and polyester bandages with casein microspheres encapsulating the TTCL. In Figure $1 \mathrm{~b}$ and $\mathrm{d}$, cotton and polyester yarns coated with relatively large spheres are presented to better illustrate the presence of microspheres on the surface of fabrics.

The efficiency of the sonochemical method in the creation and attachment of drug-loaded microspheres onto cotton and polyester fabrics was studied by using UV spectrophotometry. The sonochemistry did not destroy the TTCL, as evidenced by the very small changes in the TTCL concentration $(2-3 \%)$ that occurred during the sonication of an identical precursor solution sonicated for the same time in the absence of the BSA/casein protein. No residues of the antibiotic were found in the excess dodecane (the upper phase).

To find the optimal concentration of TTCL in the PMs, coated fabrics were prepared by using different concentrations of the drug in the precursor solution with the same concentration of protein. These studies showed that the optimal TTCL concentration in the precursor solution for microsphere attachment to cotton and polyester fabrics is $5.4 \times$ $10^{-3} \mathrm{M}$. At this concentration fabrics were obtained with PMs that contained the maximum amount of TTCL. Although the percentage of the drug (TTCL in PMs) increased with increasing concentration of TTCL in the precursor solution, it reached a maximum when the concentration of the TTCL was $5.4 \times 10^{-3} \mathrm{M}$.

The percentage of the drug in the microspheres attached to the surface of the fabric decreased with further increases of its concentration in the precursor solution. Reaching such a maximum can be explained as follows: The organic solvent is the major liquid found inside the newly formed microsphere. The solubility of TTCL in dodecane is limited and saturation occurs when this limit is reached. Although some water can also be found in the microsphere its amount is small and does not increase the amount of encapsulated TTCL.

The concentration of TTCL was then determined. After the long heating process ( 4 days at $50{ }^{\circ} \mathrm{C}$ ), 26.9 and $31.8 \%$ of TTCL were found on the cotton and polyester fabrics, respectively. These amounts include not only TTCL encapsulated in the microspheres, but also the amount of free TTCL directly adsorbed onto the cotton and polyester bandages. The direct adsorption of TTCL onto the cotton and polyester bandages was determined as described in the Experimental Section. Only 7.5 and $11.4 \%$ of TTCL were directly adsorbed onto the cotton and polyester bandages, respectively. The higher adsorption of the TTCL antibiotic onto the polyester fiber can be explained by the hydrophobic nature of polyester yarn. Both polyester and the TTCL antibiotic contain aromatic rings and hydroxy and carbonyl groups; this can produce hydrophobic interactions and hydrogen bonding. On the other hand, cotton fiber (cellulose) contains no aromatic rings; this makes the fiber less hydrophobic and more hydrophilic.

To find the exact amounts of TTCL inside the microspheres attached to the fabrics we subtract the amount of TTCL directly adsorbed onto the fabrics from the total amount of TTCL found on the surface of the fabrics. The same calculation was repeated after laundering the coated fabrics as described in the Experimental Section. The results of the calculations are presented in Table 1 .

The results show that the loss of TTCL in this "laundering" process is 5.6 and $7.8 \%$ for the cotton and polyester fabrics, respectively. If we take into account that most of the TTCL removed is pristine TTCL, we can conclude that the microspheres are strongly bonded to these fabrics and perhaps can sustain a few washing-machine cycles. In addition we conducted the following control experiment: The adsorption of TTCL-loaded spheres (PMs with TTCL inside) onto the surfaces of the fabrics was studied without sonication. The cotton and polyester fabrics were incubated with the solution of TTCL-loaded PMs for $24 \mathrm{~h}$ and then washed several times with distilled water. The drug-loaded PMs were not found attached to the surfaces of the fabrics and only around $2.6-3 \%$ of TTCL was directly adsorbed onto the surfaces of the fabrics. The results indicate that drug-loaded PMs can be attached to the fabrics only when ultrasonic radiation is applied.

We also examined the amount of protein attached to the textiles by spectrophotometric analysis $(280 \mathrm{~nm})$. First, the 
Table 1. Calculation of the amounts (\%) of TTCL encapsulated in PMs attached to the surfaces of fabrics. ${ }^{\text {[a] }}$

\begin{tabular}{|c|c|c|c|c|c|c|}
\hline Type of fabric & $\begin{array}{l}\text { Total amount of } \\
\text { TTCL on the } \\
\text { surface }[\%]\end{array}$ & $\begin{array}{l}\text { TTCL adsorbed } \\
\text { on the fabric } \\
{[\%]}\end{array}$ & $\begin{array}{l}\text { TTCL encapsulated } \\
\text { in PMs attached } \\
\text { to the fabric }[\%]\end{array}$ & $\begin{array}{l}\text { TTCL adsorbed } \\
\text { after the "laundering } \\
\text { test" [\%] }\end{array}$ & $\begin{array}{l}\text { TTCL encapsulated } \\
\text { in PMs after the } \\
\text { "laundering test" [\%] }\end{array}$ & $\begin{array}{l}\text { Loss of TTCL } \\
\text { during the "laundering" } \\
\text { process }^{[\mathrm{b}]}[\%]\end{array}$ \\
\hline cotton & 26.9 & 7.5 & $19.4 \pm 2$ & 3.7 & $17.6 \pm 1.8$ & 5.6 \\
\hline polyester & 31.8 & 11.4 & $20.4 \pm 2$ & 5.1 & $18.9 \pm 1.8$ & 7.8 \\
\hline $\mathrm{TTCL}^{[\mathrm{c}]}[\%]$ & 4.3 & 1.2 & 3.1 & 0.6 & 2.8 & 0.9 \\
\hline $\operatorname{TTCL}^{[\mathrm{d}]}[\%]$ & 8.5 & 3 & 5.4 & 1.4 & 5 & 2 \\
\hline
\end{tabular}

[a] The weights of pristine cotton and polyester fabrics were 0.15 and $0.09 \mathrm{~g}$. The initial concentration of TTCL was $5.4 \times 10^{-3} \mathrm{M}$. [b] The loss of TTCL during the "laundering process" was determined by measuring the amount of TTCL in the laundering solution after the laundering process. [c] Concentration per weight of cotton fiber. [d] Concentration per weight of polyester fiber.

amount of protein (BSA or casein) left in the aqueous solution after sonication was subtracted from the total amount of protein that was introduced. The results indicate that the total amount of protein converted into microspheres is around $87 \%$ for cotton (w/w weight ratio of protein/cotton fiber $=2.9 \%)$ and $89 \%(\mathrm{w} / \mathrm{w}$ weight ratio of protein/polyester fiber $=5 \%)$ in the case of polyester fabric in the reaction cell, which is composed of PMs bonded and nonbonded to the fabric. To determine the amount of protein bonded to the coated fabrics, we placed the fabrics in water in a glass vial and heated the solution for 4 days at $45-50{ }^{\circ} \mathrm{C}$. Heating the solution completely destroyed the microspheres. The results show that $34.8 \%(1.9 \% \mathrm{w} / \mathrm{w})$ of the BSA PMs and $47.3 \%(2.6 \% \mathrm{w} / \mathrm{w})$ of the casein protein PMs were anchored on the polyester fabric and $43.5 \%(1.45 \% \mathrm{w} / \mathrm{w})$ of BSA PMs and $31.6 \%(1 \% \mathrm{w} / \mathrm{w})$ of casein PMs were attached to the cotton fabric.

Note that when four pieces of $5 \times 5 \mathrm{~cm}$ cotton or four pieces of polyester bandages were sonicated with the precursor solution, all four pieces were coated with PMs and the amount of protein on the surface of each piece of fabric was found to be $20-22 \%(0.6-1.2 \% \mathrm{w} / \mathrm{w})$. Thus, we found that a maximum amount of four bandages could be coated simultaneously in our $50 \mathrm{~mL}$ sonication cell.

The kinetics of TTCL-antibiotic release was studied to determine the drug-delivery rate by following the changes in the TTCL UV absorption ( $366 \mathrm{~nm})$ as a function of time. The studies were performed at 25 (room temperature) and $37^{\circ} \mathrm{C}$ (body temperature). The results of the kinetic studies show that at room temperature the destruction of microspheres and TTCL release start only after 90 days and only after 280 days were $100 \%$ of the microspheres destroyed and the encapsulated TTCL released (Figure 2). At $37^{\circ} \mathrm{C}$, $100 \%$ of the antibiotic was released within 15 days.

The average sizes and electrical charges of the PMs formed in the presence of cotton and polyester fabrics in the reaction cells were determined by dynamic light scattering (DLS) measurements. The average size and the electrical charge of TTCL-loaded BSA are $1282 \mathrm{~nm}$ and $-15.7 \mathrm{mV}$, respectively. When pristine BSA or casein proteins were sonicated the DLS measurements showed spheres with an average size of $2340 \mathrm{~nm}$ for BSA PMs and $2600 \mathrm{~nm}$ for casein PMs, and an electrical charge of $-35 \mathrm{mV}$ was measured for both types of microspheres. The differences in the sizes and electrical charges of the pristine and drug-loaded PMs can

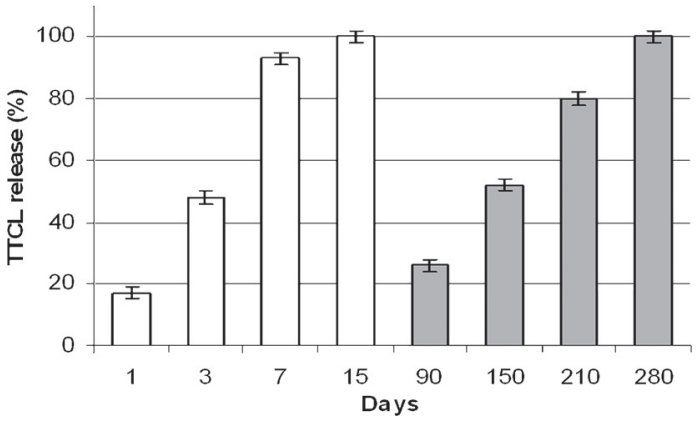

Figure 2. Kinetic studies of TTCL-antibiotic release as a function of time. The studies were performed at 25 (gray bars) and $37^{\circ} \mathrm{C}$ (white bars). At $25^{\circ} \mathrm{C}$ the release of TTCL started after 90 days and $100 \%$ of TTCL was released after 280 days. At $37^{\circ} \mathrm{C}$ the release of antibiotic started immediately and was completed within 15 days. The difference in the kinetics of TTCL release for BSA and casein proteins is $1.5-2 \%$.

be explained by the influence of TTCL on the formation of the microspheres. The electrical charge is lower in the drugloaded PMs due to the presence of TTCL molecules on the outer surfaces of the microspheres. The next step was to determine the influence of the nature of the fabric on the size and electrical charge of the created microspheres. The average size distributions of the spheres formed in the reaction cells with cotton and polyester fabrics are 1328 and $680 \mathrm{~nm}$, respectively. The electrical charges of the PMs formed in the reaction cells with cotton and polyester fabrics are -13.1 and $-8.16 \mathrm{mV}$, respectively. Although the cotton fabric exhibits PMs that are very similar in size and electrical charge to regular PMs prepared without a fabric in the sonication cell, the size and electrical charge are drastically reduced for the PMs anchored to the polyester fabric. This dramatic difference in the size of the PMs on the two fabrics was detected for BSA as well as casein and may be explained by the fact that a higher concentration of PMs is found on the polyester fabric due to hydrophobic interactions between the protein and polyester fibers. The many seeds distributed on the fabric continue to grow and because the amount of protein is spread over a larger number of seeds, smaller PMs are formed. The decrease in electrical charge from -15.7 (TTCL-loaded PMs formed in the absence of polyester in the reaction cell) to $-8.16 \mathrm{mV}$ can be explained by the more important role of the TTCL molecules on the smaller spheres formed on the polyester fabric leading to a larger 
decrease from the value of $-35 \mathrm{mV}$ detected for pristine PMs.

The antimicrobial activities of the TTCL-loaded BSA and casein microspheres attached to cotton and polyester bandages was tested on two bacterial strains (Staphylococcus aureus and Escherichia coli) that are sensitive to TTCL. The results are summarized in Table 2.

Table 2. Inhibition zones for PM-coated fabrics (TTCL-loaded PMs) and a commercial tablet of TTCL. ${ }^{[a]}$

\begin{tabular}{lcll}
\hline Type of material & TTCL $[\mu \mathrm{g}]$ & $\begin{array}{l}\text { Inhibition zone } \\
\text { S. aureus }[\mathrm{mm}]\end{array}$ & $\begin{array}{l}\text { Inhibition zone } \\
\text { E. coli }[\mathrm{mm}]\end{array}$ \\
\hline Commercial tablet of TTCL & 30 & 27 & 19 \\
Cotton with TTCL-loaded PMs & 12 & 27 & 18 \\
Polyester with TTCL-loaded PMs & 12 & 26.5 & 17 \\
Clean cotton fabric & 0 & 0 & 0 \\
Clean polyester fabric & 0 & 0 & 0 \\
\hline
\end{tabular}

[a] The antibacterial activity was measured for coated fabrics after the "laundering" process.

Bandages coated with microspheres loaded with TTCL show an inhibition zone similar to that of a commercial tablet of TTCL (Figure 3). The inhibition zone for the commercial tablet was $27 \mathrm{~mm}$ versus $S$. aureus and the same size zone was also observed for the PMs coated on cotton. For E. coli the commercial tablet revealed an inhibition zone of $19 \mathrm{~mm}$, whereas the PMs coated on cotton showed an inhibition zone of $18 \mathrm{~mm}$. The inhibition zone for the coated polyester was $26.5 \mathrm{~mm}$ for $S$. aureus and $17 \mathrm{~mm}$ for $E$. coli. The TTCL in the commercial tablet and inside the microspheres attached to the surfaces of the fabrics was 30 and approximately $12 \mu \mathrm{g}$, respectively. Thus, the coated PMs show a stronger killing affect towards these two bacterial strains.
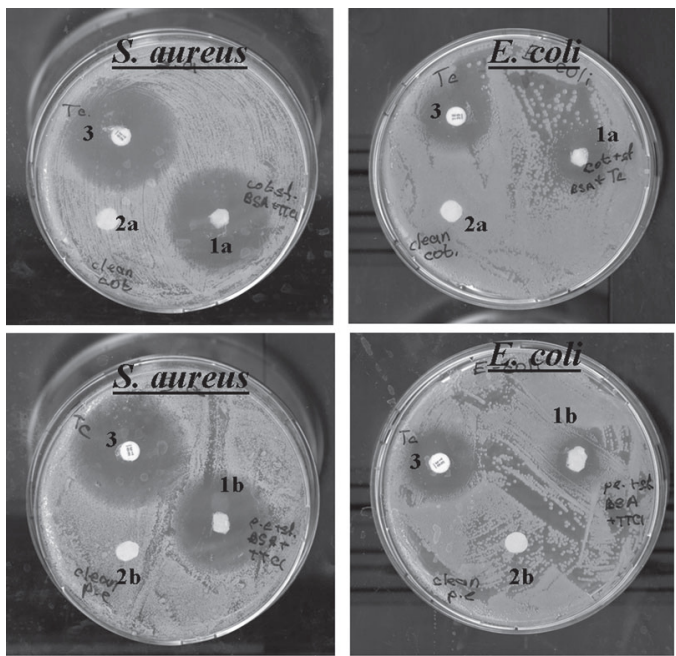

Figure 3. Inhibition zones of $S$. aureus and E. coli on agar plates as a result of 1a) TTCL freed from microspheres attached to cotton fabric, 1b) TTCL freed from microspheres attached to polyester fabric, 2a) clean cotton fabric, 2b) clean polyester fabric, and 3) commercial tablet of TTCL.
It seems that the TTCL trapped in the microspheres attached to the surfaces of the cotton and polyester fabrics and released to the media is as active as TTCL freed from the commercial tablet. Nevertheless, the "inhibition-zone" results indicate that the encapsulated TTCL "antibacterial" effect is superior to that of the commercial tablet because a third of the amount of TTCL is needed in the coated fabrics to reach the same antimicrobial activity as that of the commercial TTCL. Note that the TTCL is released in a fast mode due to the protease activity.

\section{Conclusion}

By using sonochemical radiation we have succeeded in attaching drug-loaded proteinaceous (BSA and/ or casein) microspheres to cotton and polyester fabrics in a one-step process that lasts just a few minutes. We have found that if during the sonication time a drug and a piece of fabric are added to the precursor mixture containing an aqueous solution of a protein plus an overlaying organic solvent, the drug will be encapsulated inside the microspheres and these microspheres will bond to the fabric. The average size of microspheres anchored to cotton and polyester fabrics are $1328 \mathrm{~nm}$ for cotton and $680 \mathrm{~nm}$ for polyester. This can be explained by the differences in the chemical structures of the fabrics. The results suggest that the coated fabrics can be used as antibacterial fabrics. Coated bandages revealed an antibacterial activity stronger than that of a commercial TTCL tablet.

To summarize, the sonochemically treated bandages of cotton and polyester coated with microspheres loaded with TTCL were found to be active as antimicrobial agents.

\section{Experimental Section}

Attaching microspheres containing drugs to fabrics by using ultrasound radiation: To attach BSA or casein microspheres containing TTCL to cotton or polyester fabrics, dodecane $(6.7 \mathrm{~mL}, 98.0 \%$ Fluka) was layered over aqueous BSA (albumin, bovine fraction v, Sigma; $10 \mathrm{~mL}, 5 \% \mathrm{w} / \mathrm{v}$ ) or casein (casein sodium salt from bovine milk, Sigma-Aldrich) solution with a piece $(5 \times 5 \mathrm{~cm})$ of cotton or polyester fabric. TTCL $(0.024 \mathrm{~g})$ was added to the above-mentioned precursor mixture (to the aqueous solution of $5 \%$ BSA). The weights of the pristine cotton and polyester fabrics were 0.15 and $0.09 \mathrm{~g}$.

The solution was sonicated for 3 min with a high-intensity ultrasonic probe (Sonic and Materials, VC-600, $20 \mathrm{kHz}, 0.5$ in a Ti horn at $30 \%$ amplitude). The bottom of the high-intensity ultrasonic horn was positioned at the aqueous/organic interface employing an acoustic power of approximately $58 \mathrm{~W} \mathrm{~cm}^{-2}$ with an initial temperature of $22^{\circ} \mathrm{C}$ in the reaction cell. An ice-cooling bath was used to keep the temperature constant. At the end of the reaction, the bandage was washed with water $(3 \times 5 \mathrm{~mL})$ to remove the residue of the unbound microspheres and the pristine protein molecules.

Determination of the TTCL concentration in the BSA/casein microspheres bonded to the fabrics: The distribution of the total amount of tetracycline (encapsulated, not encapsulated, and the residue in the solution) was determined by absorption measurements as explained below. 
The UV studies on TTCL were conducted with a Carry 100 spectrophotometer at $366 \mathrm{~nm}$. The calculations were based on the Beer-Lambert law: $A=\varepsilon c l$ (the TTCL molar extinction coefficient, $\varepsilon$, is $14.15 \times$ $10^{-3} \mathrm{~L} \mathrm{~mol}^{-1} \mathrm{~cm}^{-1}$ ). The calculations were performed as follows: The absorption of individual TTCL molecules (not encapsulated in the microspheres) removed from the fabric surface was measured. The free molecules were removed by soaking the coated fabric in water for $24 \mathrm{~h}$. The amount of TTCL loaded in the microspheres attached to the fabrics was measured by sonicating the fabric after $24 \mathrm{~h}$ of soaking. The sonication was performed for $3 \mathrm{~min}$ and no microspheres were found on the fabric when it was analyzed under the microscope. The microspheres were destroyed and the amount of TTCL in the microspheres was then determined. By adding the amount of "free" drug (TTCL that was not encapsulated in the microspheres attached to bandage), the amount of encapsulated TTCL, and the amount remaining in the aqueous solution after the sonication, we should obtain the total amount of TTCL used in the coating process. The TTCL distribution was also determined by placing the cotton or polyester bandage in a glass vial with water $(5 \mathrm{~mL})$ and heating the liquid for 4 days at $50^{\circ} \mathrm{C}$. Because the long heating process completely destroyed the microspheres attached to the fabric, the free TTCL and the TTCL in the microspheres could be determined.

Determination of the amount of protein bonded to the fabric: The amount of protein (BSA and casein) bonded to the bandages was measured by using a NanoDrop 1000 spectrophotometer at $280 \mathrm{~nm}$. The amount of protein attached to the fabric was determined by subtracting the amount of "free" protein (protein in microspheres that are not attached to bandages) and the amount of protein in the residue phase (the lower phase in the separation flask) from the total amount in the precursor solution.

Washing test: To remove impurities or unattached microspheres from the cotton and polyester fabrics, the samples were washed with $2 \mathrm{gL}^{-1}$ of a nonionic agent, Lutensol AT $25\left(10 \mathrm{gL}^{-1}\right)$ for $40 \mathrm{~min}$ at $60^{\circ} \mathrm{C}$. The laundering test was performed on a rotor wash machine under the following conditions: $60^{\circ} \mathrm{C}, 90 \mathrm{~min}, 40$ cycles per minute.

Antimicrobial activity of coated fabrics: The antimicrobial activity of the bandage with TTCL-loaded BSA microspheres was tested on two bacterial strains that are sensitive to TTCL. One strain, S. aureus (101), represents Gram positive bacteria and the other, E. coli (1313), represents Gram negative bacteria. Each of the strains was spread on nutrient agar plates and the bandage to be tested (diameter $=5 \mathrm{~mm}$ ) was put on seeded plates for $24 \mathrm{~h}$ at $37^{\circ} \mathrm{C}$

Characterization methods: DLS and zeta-potential measurements were carried out on an ALV/CGS-3 compact goniometer system equipped with an ALV/LSE-5003 light-scattering electronic and a multiple s digital correlator, and a $632.8 \mathrm{~nm}$ JDSU 1145P laser. DLS and z-potential experiments were carried out on a doubly diluted as-separated PM solution, that is, the PMs removed after sonication were diluted with an equal amount of doubly distilled water. Each measurement took $10 \mathrm{~s}$; particle distribution and electrical charge distribution were obtained by averaging 10 DLS measurements.

For light microscopy (Apo-Tome Microscope, Zeiss), samples were prepared by depositing aqueous dispersions, without further purification, on a glass-slide.

\section{Acknowledgements}

This research was carried out as part of the activities of the LIDWINE Consortium, Contract No. NMP2-CT-2006-026741. LIDWINE is an IP Project of the 6th European Customers (EC) Program.

[1] G. Sun, X. Xu, J. R. Bickett, Ind. Eng. Chem. Res. 2001, 40, 10161021.

[2] W. Whyte, R. Hodgson, B. V. Bailey, J. Graham, Br. J. Surg. 1978, $65,469-474$.

[3] A. N. Neely, M. P. Maley, J. Clin. Microbiol. 2000, 38, 724-726.

[4] S. F. Bloomfield, E. Scott, J. Appl. Microbiol. 1997, 83, 1-9.

[5] A. Hambraeus, J. Hyg. 1973, 71, 787-797.

[6] T. L. Vigo, M. A. Benjaminson, Text. Res. J. 1981, 51, 454-465.

[7] J. S. Cho, G. Cho, Text. Res. J. 1999, 69, 875-880.

[8] T. L. Vigo, Biotechnology and Bioactive Polymers (Eds.: C. Gebelein, C. Carraher), Plenum Press, New York. 1994, pp. 225-237.

[9] A. J. Isquith, E. A. Abbot, P. A. Walters, Appl. Microbiol. 1972, 24, 859-863.

[10] J. L. Speier, J. R. Malek, J. Colloid Interface Sci. 1982, 89, 68-76.

[11] B. Mahltig, D. Fiedler, A. Fischer, P. Simon, J. Sol-Gel Sci. Technol. 2010, 55, 269-277.

[12] D. Hoefer, T. R. Hammer. ISRN Dermatology, Article ID 369603, 8 pages, 2011.

[13] I. Perelshtein, G. Applerot, N. Perkas, G. Guibert, S. Mikhailov, A. Gedanken, Nanotechnology 2008, 19, 245705.

[14] I. Perelshtein, G. Applerot, N. Perkas, E. Wehrschetz-Sigl, A. Hasmann, G. M. Guebitz, A. Gedanken, Appl. Mater. Interfaces 2009, 1, 361-366.

[15] U. Angel (Shimanovich), C. M. Silva, A. Cavaco-Paulo, A. Gedanken, Isr. J. Chem. 2010, 50, 524-529.

[16] A. Gedanken, Chem. Eur. J. 2008, 14, 3840-3853.

[17] M. Wong, K. J. Kolbeck, K. S. Suslick, Abstr. Pap. Am. Chem. Soc. 1994, 207, 79-COLL.

[18] U. Angel (Shimanovich), D. Matas, S. Michaeli, A. Cavaco-Paulo, A. Gedanken, Chem. Eur. J. 2010, 16, 2108-2114.

[19] E. R. Garrett, G. H. Miller, Pharm. J. 1965, 54, 427.

[20] Q. X. Yang, J. Zhang, W. Y. Zhang, Z. Wang, Y. Xie, H. Zhang, J. Environ. Sci. Health Part B 2010, 45, 190-197.

[21] Y. Han, Q. A. Chang, T. Virag, N. C. West, D. George, M. G. Castro, M. C. Bohn, Gene Ther. 2010, 17, 616-625.

[22] M. A. Kaplan, W. T. Bradner, F. H. Buckwalt, M. H. Pindell, Nature $\mathbf{1 9 6 5}, 205,399$

Received: March 14, 2011

Revised: July 20, 2011

Published online: November 30, 2011 